\title{
EDITORIAL
}

\section{Game over for sports cheats?}

T he 2010 Fédération Internationale de Football Association (FIFA) World Cup has just been held in South Africa. Even before the tournament kicked off, eight players from each of the 32 qualifying teams were randomly selected to undergo compulsory, 'without notice' drugs testing; additional tests were performed after each match during the month-long competition. Was this crackdown by FIFA overkill or simply a sad reflection on the current state of elite sports?

The FIFA World Cup historically has a good track record on doping. However, the use of performanceenhancing drugs such as anabolic androgenic steroids and recombinant human growth hormone is now prevalent in many competitive sports, including baseball, cycling and athletics. The reason why some athletes are tempted to cheat is no great mystery: the winner really can take it all. Nevertheless, cheating is not without risk. The misuse of agents designed primarily for therapeutic purposes can cause serious (and sometimes fatal) adverse effects. Furthermore, athletes who are caught cheating are liable to hefty fines, suspension and public vilification. Despite these penalties, many cheats believe they can get away with it-but the past decade has witnessed a major shift in the response to drug abuse in sports.

The World Anti-Doping Agency (WADA) is the frontline in the war on sports cheats. WADA was established in 1999 to promote, coordinate and monitor international anti-doping strategies by working closely with government agencies and sporting associations like FIFA. A key function of WADA is to support the development of assays to detect performance-enhancing drugs and the accreditation of a global network of anti-doping laboratories. In addition, WADA publishes an annual prohibited substances list; endocrine-related drugs feature heavily on this register.

WADA recently rolled out a groundbreaking new initiative: the athlete biological passport. In addition to the usual random drugs tests, athletes are now required to undergo regular monitoring of biological variables, such as their levels of hemoglobin or red blood cell count. Plotting these measurements over time and looking for abnormal variations should facilitate indirect detection of doping because the downstream effects often remain evident long after the actual drug has disappeared from the body. On the face of it, longitudinal screening is an excellent idea, but only time will tell whether the athlete biological passport proves an effective deterrent.
At present, the passport is restricted to eight hematological markers. Rapid expansion of this panel to include measurements of endocrine function should be a priority as many of the banned drugs target the endocrine system. The potential for gene doping must also be explored.

As an organization, WADA has made great strides in anti-doping policy over the past 10 years. What part can the individual play in making sport drugs-free? Rather than leaving it all to the bureaucrats, a grassroots approach to the problem should be encouraged, with the aim of preventing drug abuse from happening in the first place. The use of performance-enhancing drugs is not restricted to elite athletes; published data suggest that abuse of anabolic androgenic steroids and other endocrine drugs is on the rise among high-school and college athletes. Therefore, it is crucial that young people and their mentors are properly educated about the risks involved. Clinicians and other health-care professionals should take the initiative in this respect, as they have a duty of care to highlight the uncertain benefits and potentially harmful effects of doping. Medical practitioners should also familiarize themselves with the contents of the WADA prohibited substances list, including the rules on in-competition versus out-of-competition drug use, and the correct procedure for therapeutic use exemption. Finally, clinicians must have the courage to examine their own culpability when drugs violations are committed by athletes under their care.

Some people say that attempting to combat doping in sports is a waste of time and resources. Proponents argue that scrapping the WADA prohibited substances list will give everyone the same 'opportunities' and create a level playing field. But such a stance misses the point. Aside from the health-related consequences of drug abuse, sport has a key societal role, with the potential to literally change lives. In 1995, South Africa hosted (and won) the Rugby World Cup. The image of Nelson Mandela presenting the trophy to François Pienaar, the white captain of the South African team, represented a pivotal moment in the breakdown of apartheid. Furthermore, participation in sport fosters many admirable qualities: dedication, discipline, leadership, teamwork, self-esteem and a sense of community. Doping tarnishes these ideals. It is time to blow the final whistle on sports cheats and champion fair play.

doi:10.1038/nrendo.2010.120

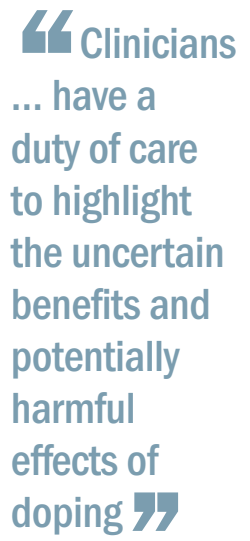

Vicky Heath is the Chief Editor of Nature Reviews Endocrinology.

Competing interests The author declares no competing interests. 